\title{
At the zoo
}

\section{The last two baseline humans in captivity have bred successfully.}

\section{2}

Warren Ellis 8/03/2468 2225.18 Universal Time Code. The Human Reservation, in temperate London, England, considers the breeding of the last two baseline humans a vindication of its hard work in identifying, isolating and protecting the 'pure', secondmillennium strain of human being.

"The cusp between second and third millennium is vital," explained the baseline humans' head keeper, Daybreak Sigridsdottir, in a brief radiotelepathy interview conducted during the act of disposing of the couple's physical waste products, known as 'mucking out'. "The emergence of practical biotechnology happened right at that point. The introduction of artificial and alien material into the human genetic structure began barely ten years into the new millennium. You can't trust anything after 2001."

Sigridsdottir is an accomplished zoologist, whose text about the rescue and subsequent massive sexing of the population of New York City after the release of the Genital Babel terrorist bioware won her the William Gates VI Truth Prize for 2461. Her work was crucial in sifting through world records to locate humans who remained utterly unchanged by modern biological technology. She well remembers the conflict of feelings when it was discovered that only two humans on Earth still possess a pre-2000 biological make-up.

"We stopped thinking about the way we live a long time ago. We take a deep breath to oxygenate enough for winged flight, but we don't consider what we left in that lungful of air. In 66 cases out of a hundred, a cubic metre of sea-level air will contain an airborne genetic trait, engineered in a lab or spored from a working organ, capable of applying itself to the biology of the respiring individual.

"And now here we are. Most of us are at least $0.5 \%$ of our DNA away from the human baseline. Some are more. On the other side of the human species, if you go a percent or two of DNA away, you have a chimp. By the same token, many of us are no longer human. We are something else entirely. And the horror and surprise and sadness that has been expressed are artefacts of our refusal to accept that."

Nine Nevada Rockets, spokesgroup for the Transhuman Association, relays a different perspective on the event from its spawning territory in the Pacific Ocean. "This is nonsense. I could make a case for the genome being 'untrustworthy' post-1945, with the introduction of trace elements from nuclear detonations into the environment. Human genetic structure changed with each new immunity we developed and passed down to our descendents. Who is the Human Reservation to say what is human and what isn't?"

Sigridsdottir, who points out that Rockets is responding via a telepathy organ derived from whale and eel biologies, responds: "Look at us. We've adopted mechanical traits, we've taken on animal traits, we've invented new abilities and new internal organs to perform them from scratch. Rockets is nine neo-dolphin bio-

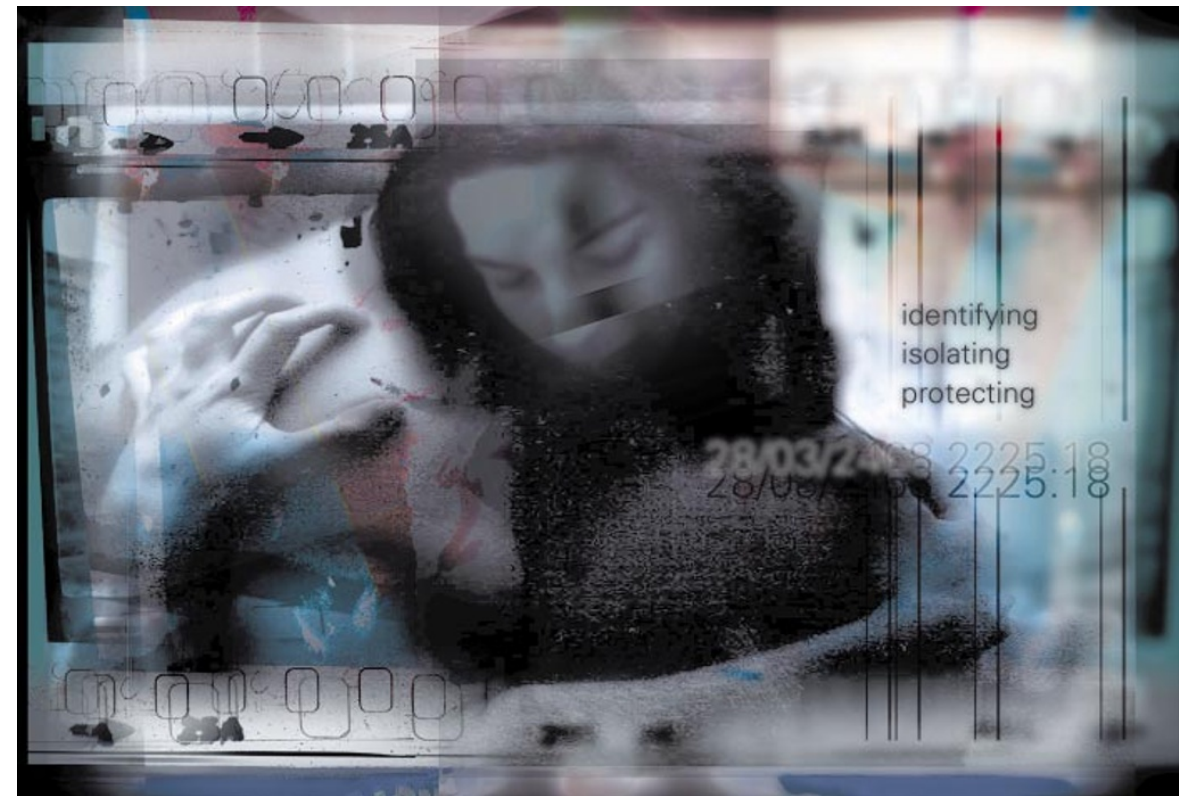

forms sharing a single brain on a local-access network run by organic modems located in their beaks. We are as far removed from these two people as they are from chimpanzees in the wild."

The unnamed couple, existing in a large and heavily secured enclosure dressed to resemble the urban environment they were found in, have not had an easy captivity. They have endured terrorist attempts to corrupt their genome, abortive assassination strikes, and even bomb threats.

One suspect in the latter is Laura Magdelene Manson, amanuensis for the nondenominational Assembly Of Mysterious Devotion, a barely visible cloud of femtotechnological machinery: "We were created to adapt and change. It was our appointed role to take on the traits of animals - to contain within ourselves all the abilities of the machines we have conceived. We do this to become worthy companions of The Mystery. To propagate and preserve inferior iterations of the human being is sick."

Sigridsdottir is dismissive. "It's all part of the ongoing psychosis of the culture. Despite the fact that we take for granted physical abilities that the second-millennium human could find only in outré fiction, we somehow feel threatened by the notion that someone is 'purer' than us. And the Assembly hasn't been the same since it was banned from using the word 'God' and other inflammatory antique terms. Preservation is a vital function of a civilized culture."

Why was physical, 'classic' breeding required? "Because it's now almost impossible to guarantee the absolute purity of a laboratory environment. And even if we could clone them in complete protection - that kind of defeats the point, to me. Using the classic method seems to me to be the most aesthetic way to accomplish this. It's the organic way. And, personally, I found it fascinating. No-one in my family has had actual, traditional, x penetrative sex in seven generations. And now I see why."

So how difficult was it to implement the breeding? "Well, it wasn't as easy as you'd think. Sperm counts were remarkably low in the late-twentieth-century human male. The genetic imperative to reproduce was slowing over much of the world due to the rise in live births and changes in societal mores. Frankly, we had to get her drunk and promise him a pizza."

Warren Ellis (http://www.warrenellis.com) is the author of the award-winning Transmetropolitan series of graphic novels and various other works in the comics field. 